\title{
Blockchain Economics and Marketing
}

\section{Frank T. Lorne, Sriram Daram, Rutha Frantz, Naveen Kumar, Athif Mohammed, Amit Muley}

School of Management, New York Institute of Technology, Vancouver, Canada

Email: florne@nyit.edu

How to cite this paper: Lorne, F.T., Daram, S., Frantz, R., Kumar, N., Mohammed, A. and Muley, A. (2018) Blockchain Economics and Marketing. Journal of Computer and Communications, 6, 107-117. https://doi.org/10.4236/jcc.2018.612011

Received: September 17, 2018 Accepted: December 23, 2018 Published: December 26, 2018

\begin{abstract}
Blockchain as a supporting platform for various cryptocurrencies is a technology that will not sell by itself, meaning that its success could not be built on technical efficiency of an algorithm alone. Understanding the real economic problems that the technology should aim at solving is a first step towards identifying the marketing beachheads for the technology. The branding of any blockchain can be categorized under an economic framework pointing to the types that have the potentials of being sustainable and disruptive. Selection of appropriate beachheads, verticals, services etc. should aim to promote the exchange of real goods and services, and/or the utilization the accounting/data advantage of the technology.
\end{abstract}

\section{Keywords}

Transaction Costs, Cryptoeconomics, Marketing

\section{Blockchain Economics}

Blockchain is the backbone of every cryptocurrency [1]. As such, blockchain goes beyond a distributed ledger accounting system, which usually involves the adoption or the invention of a particular cryptocurrency [2]. Cryptocurrency became a well-recognized name since bitcoin achieved popularity and a rapid increase in value (as well as fluctuation) [3] [4]. As a substitute for money, a cryptocurrency must satisfy three fundamental functions of money [5]: 1) as a medium of exchange, 2) as units of accounts (measuring wealth and transactions), 3) as a store of value. Studies of adoption rate of cryptocurrency undoubtedly refer to these three fundamental functions for formulating a framework for their analyses [6]. However, many economists have expressed doubts on whether cryptocurrencies can ever replace money. ${ }^{1}$

${ }^{1}$ See for example https://www.nytimes.com/2018/01/29/opinion/bitcoin-bubble-fraud.html,https://www.ccn.com/topeconomists-stiglitz-roubini-and-rogoff-renew-bitcoin-doom-scenarios/, https://www.forbes.com/sites/jemmagreen/2018/07/25/blockchains-and-smart-contracts-whos-really -in-charge/\#3b2488b47854. One went far by calling this to be The Big Blockchain Lie, and the mother of all bubbles. 
Anticipating the ever-expanding digital commerce on the internet, it is a matter of time that more and more transactions will be carried out digitally, over-the-wire or wirelessly. With a forever evolving generation of young replacing the old, and together with that, an evolving changing of lifestyle, it is not too far-fetched to believe that new methods of exchange can displace conventional payment methods on the internet, and eventually, spilling over to the brick-and-mortar life. It is by accepting this scenario as a real possibility in future lifestyle that it would make sense for economists to consider cryptocurrencies seriously.

Currently, various cryptocurrencies differ in their usefulness in the three functions of money. Economic theories could suggest that the first two functions are the most important functions of money in its ability to reduce transaction costs of bartering, implying that when the values of the first two functions are identified and secured, the third dimension, i.e. the store of value, will automatically follow. This premise is based on the belief that any disruptive innovation must ultimately solve real economic problems in business and relational transactions, rather than it being innovative just as a new mathematical algorithm. Mathematics, as a stand-alone product, is pure elegance. The value of elegance is metaphysical. Thus, if the branding of a blockchain technology focuses only on the third dimension alone, e.g. hypes on a rapid increase in the currency value and that feature only, it will not necessarily lead to values being created in the first two functions. Indeed, persuasive arguments can be provided that risky value assets will mitigate its true intrinsic usages, as people are averse to volatility.

Liquidity risk is intimately related to the function of money as a store of value. Store of value would be for short run or for long run purposes. For a short run store of value, it could be speculative in nature, thus being negative both in terms of investment performance as well as suffering losses in new endeavors. For long run store of value, the holding of a currency cannot be justified based on past records, just like gold cannot be justified being held as a store of value solely based on the price of gold in the past. Ultimately, the long run value of a cryptocurrency depends on the usefulness of the first two functions, on how extensively a particular cryptocurrency is being used for the trading of real goods and services, and equally important, how the central banks of the world are controlling the supply of their respective currencies, regulations adopted in countries, and their attitudes and policies toward emerging technologies that can threaten countries' fiat currencies. All these factors can contribute to the success for particular blockchain usage in particular applications, for specific countries as well as for the world market.

Blockchain technology usually exhibits an algorithm enabling a fixed supply of the cryptocurrency it supports; as such, it avoids the contamination of arbitrary monetary policies of central banks. It is often on this premise that cryptocurrencies are viewed as a better method of insuring its value over fiat money. Advocates of cryptocurrencies argue that money in the bank is NOT safe, and 
that owning some type of cryptocurrencies in the portfolio is the ONLY self-sovereignty of wealth. This argument, however, is valid only if the cryptocurrency being held can fulfill the other two functions of money. If not, a cryptocurrency as a store of value is similar to a penny stock's worthiness as a store of value. The penny stock may make an investor get rich quickly, but its value can also drop to zero if the company issuing the penny stock has no real economic products and services that can contribute to the intrinsic value for the stock. Thus, creating a currency purely for the sake of generating store of values will not necessarily insure a self-sovereignty of wealth. In addition, governments can still confiscate cryptocurrencies owned by a person simply by forcing the person to surrender the key of an account. Therefore, branding a particular blockchain technology purely as fulfilling a better function to store values may not be the best focus.

If blockchain technology wants to achieve its potentials as a transaction costs reduction mechanism for decentralized transactions, it should focus on the first two functions of money. The medium of exchange function of money focuses on how a particular blockchain technology can reduce the transaction costs of exchanges. The intrinsic nature of transaction costs has been vastly studied in economic literature; and often, it is intrinsic nature that gives rise to middlemen who can help facilitate exchanges [7]. These exchanges, often are for financial assets, but also can be for real goods and services, resulting in transaction (middlemen) fees that can be very high. To address to whether a particular blockchain technology can reduce transaction costs, it is necessary that both the intrinsic nature of transaction costs as well as middlemen's market transaction fees are being studied.

The intrinsic nature of transaction costs encompasses two types: adverse selection and moral hazard. Adverse selection is a pre-contracting risk, typically a lemon problem [8], but also referring to a situation where the offer of goods and services attracting customers that the sellers precisely wish to avoid. Moral hazard is a post-contracting risk, referring to a situation where one party of a transaction does not take proper care of some originally understood expectations of the transaction. The origin of these types of problem arise from asymmetric information in contracting parties' identity, as well as the impossibility of fully specifying all characteristics of the real goods and services that are to be transacted. Asymmetric information is the source of what social scientists had called a "Principal-Agent problem" [9]. Inquiring how smart contracts in blockchain can help alleviate this economic problem is exceedingly important if blockchain aims to serve transactions between strangers. Inquiries about strangers could be difficult. In this respect, although Blockchain can track identity of work and persons, it cannot dig up hidden information about the work and the persons, unless it is AI enabled. Therefore, in addition to the question on whether cryptocurrency will ever duplicate the role of money and possibly replacing it as pointed out in the beginning paragraphs of this paper, we believe there are both a micro as well as a macro component to the class of problem as- 
sociated with cryptoeconomics that need to be analyzed and studied [10]. ${ }^{2}$

The heart of blockchain is to use the distributed ledger technology for witnessing decentralized private transactions. All transactions are either implicit or explicit contracts, all problems arising out of asymmetric information are transaction costs, the intrinsic transaction costs that go beyond nominal fees charged by middlemen. The fees charged reflect the service they provide in lowering the intrinsic transaction costs. The fees themselves are not transaction costs. To say that transaction costs can ever be reduced to zero is a myth. Economic Nobel Prize winner, Oliver Hart, a specialist on contract theory who earned his Prize based on a Principal-Agent problem he formulated, once expressed doubt that a smart contract can ever be made to be a cure-for-all (See fn. 1). He, however, later joined a blockchain company as their advisor in help formulating smart contracts. We are unsure at this juncture of our research whether economists' Principal-Agent problems can be sufficiently addressed by smart contracts. Our guess is that it is likely to entail some elements of artificial intelligence (AI). Regardless of emerging technologies on AI, branding of a particular blockchain technology must focus on its ability to reduce the transaction costs of goods and services exchange, whether it is more capable of making contracts "smart", or being able to circumvent traditional forms of middlemen market transaction fees.

How a particular blockchain technology can serve the second function of money, i.e. as units of accounting, is also quite important. Accounting requires creditability, which is what the consensual witness mechanism that blockchain can provide. A good consensual witness mechanism replaces the role of a judge. But is it a good judge? Does he do his job fast enough? These are questions that a smart contract should ask. It has been argued that computation power is what gives bitcoins their values. If authentication is the crux of a creditable blockchain technology, evaluating its efficiency in the authentication process is important. For example, mining of a bitcoin will on average takes 10 minutes, while an alternative cryptocurrency will on average take a different time based on its respective mining algorithm and the miners in the network of mining. This is only one aspect of efficiency, however. Related to this discussion also is whether a particular blockchain technology is open source or closed source. It has been argued that any cryptocurrency, in order to be successful, must be open source. ${ }^{3}$ This proposition is likely to be debated for some time, very much like the debate between Windows and Linux [11]. ${ }^{4}$

Another moving part of the blockchain technology is the various evolving ap-

${ }^{2}$ Cryptoeconomicscan be very broad in scope. Its relationship with AI was suggested in a presentation by Dinis Guarda on May 8, 2018 in a conference entitled The Future of Blockchain, Vancouver, B.C., Canada. We believe many of the micro transaction costs problem studied for many years in the economic literature need to be included into this area of study.

${ }^{3}$ Teeka Tiwari, Debate on Cryptocurrency vs. Gold, Vancouver Resource Investment Conference. Jan. 22, 2018.

${ }^{4}$ See the classic discussion on this in "The Cathedral and the Bazaar" on the internet http://www.catb.org/esr/writings/cathedral-bazaar/ 
plications in thee-commerce space. The branding of a particular blockchain technology might have to be evaluated in conjunction with its potentials to co-develop with these other emerging technologies. There are various types of share economies, most notably Airbnb, which 10 years ago would be considered as a very high transaction cost type of business transaction, as no one would like to share their living spaces with strangers. Now with effective screening leverage technologies, transaction costs of sharing can be reduced thereby making the living arrangement practical. If a particular blockchain technology is matched with certain sharing economies that are expanding and rising in volumes of usage and transaction worldwide, this could very well be the beachhead where blockchain technology can become disruptive. It will become disruptive if sharing becomes increasingly prevalent.

De-intermediation (elimination of middlemen) is a distinguishing feature of blockchain technology. The ultimate scenario for a blockchain technology to be entirely disruptive would replace all centralized platforms and their coordinating websites. This prediction can sometimes be misunderstood as it eventually displacing all existing institutions and brand names. That need not be the case if existing institutions and brand names, in anticipation of the disruption, embrace and adopt the technology. In that case, existing institutions and brand names essentially all operate as platforms, rather than "firms" in the traditional economic sense of the term as defined by economists [12]. From an evolutionary perspective, there is nothing unusual about this scenario, as one point far back in the history of mankind, all firms are simply a farmer working with his cow on the field.

The entry point for blockchain as a disruptive technology has yet to be played out. Irrespective of whether there will be a wholesale substitution of technology, at this stage of the technology, it seems well accepted that blockchain can indeed save transaction costs, perhaps more so in certain industries and certain applications than others. The relevant question to ask is what industries, what applications will be the beachheads, the means to cross the chasm of for the technology [13]. To this we now turn.

\section{Blockchain Verticals}

The most ubiquitous type of blockchain companies in the ecosystem of blockchain, at the time of writing of this paper, are platforms built for the purpose of currency exchange/trading. These platforms operate in principle not that different between any fiat money trading platforms except cryptocurrencies being included in the products on the platform. It would be incorrect to believe these exchanges are completely decentralized involving no service fees. Platforms still compete in terms of service fees, and it is doubtful that the fees will ever to fall to zero, as the outcome in that case will not be incentive compatible.

Currency exchange business alone, is not sufficient to build a niche that will be disruptive, just like there is no fiat money exchange business that can domi- 
nate the world market of fiat currencies. These exchanges are built on specific customer niches, reducing transaction costs for particular customer base, e.g. some aim for a game-playing base, and a promise of future interoperability between all currencies. In the long run, not any one single currency trading platform is likely to dominate a world market. There are regulatory hurdles and governance issues on "Anti-Money Laundering/Know your Customer" AML/KYC that would be standing in the way. ${ }^{5}$ Building niches of exchanges and their branding must come from additional services, possibly also in the form of hardwares. Identifying verticals, and finding ways to monetize them, is a pre-requisite for the branding of these beachheads. In this section, we describe several popular beachheads typically associated with blockchain technologies we have observed.

The most common verticals observed for blockchains fall within 4 categories: 1) Money Services and Digital Wallets, 2) Cryptocurrency Mining, 3) Capital Markets and Financial Services, 4) Merchant Services and Merchant Payments [14]. Among the four categories, we see 3 , and possibly 4 , having the highest potentials of being disruptive. Category 3 is characterized by high transaction fees while the underlying intrinsic transaction costs are not high. Transactions within a network of banks and financial institutions do not face a severe asymmetric information problem as characterized by economists' notion of transaction costs. Adverse selection with scam artists is unlikely, as not everyone can call himself/herself a bank. Institutional players have long history and creditability, and usually certified by regulatory authorities in order to be a member of a network. Excessive risks of a member also are usually curbed by regulatory authorities, thus relying on the authority or some industry self-regulating bodies to handle their moral hazard problems. In addition, there is an expectation that they are simply "too large to fail", i.e. that they will eventually be bailed out should large scale financial crisis break out. Thus, the usual type of problems associated with transactions between strangers is less of a problem for Category 3 . Focusing on financial institutions as a beachhead is not being unfaithful to the blockchain mission of decentralized exchanges, as prescreening participants to a network rather than free access open to all is only the first stage development of its adoption.

Currently, discussion around adoption of particular cryptocurrency had been centered around technical efficiency which, in the context of a broader economic framework, is only one component of a successful formula. It is often noted, for example, that Ripple can perform 1500 transactions per second (TPS), as com-

${ }^{5}$ The US Security Exchange Commission (SEC) created a Fintech Hub to help Blockchain startups to navigate ICO rules in US. Similarly, Canadian Securities Exchange (CSE) is developing a platform called Security Token Offerings (STO) that will enable companies to issue conventional equity and debt through tokenized securities. At the time of writing, a Canadian self-regulating investment community, the Investment Industry Regulatory Organization of Canada (IIROC) is creating working groups for proposals to regulate. https://cointelegraph.com/news/canada-s-investment-industry-organization-to-issue-proposals-on-b lockchain-crypto-regulations 
pared with the Ethereum doing 15 TPS and Bitcoin doing 3 - 6 TPS. Hedera Hashgraph, allegedly, can perform higher than all existing platforms. This competition in terms of efficiency certainly is important, but it is the usability, the liquidity and the volume enabled by a platform that matter more, and ultimately decides what is disruptive. In terms of impact, Ripple's XRP, it certainly has been impressive as a snapshot of this ongoing process of competition (Table 1).

The ranking of liquidity is likely to fluctuate and changing over time. The table is revealing, nevertheless, in that general public perception of cryptocurrency had been largely being bitcoins. This table shows that the more accurate picture being that the industry is forever a competition between currencies; and at any period of time, it is not a sure thing that bitcoins will evolve as the winning most circulated cryptocurrency. Banking and financial institutions, because of their needs to maintain high credibility in their business, have less of the intrinsic transaction costs problems typically among complete strangers. Platforms aiming for endorsement and adoption in Category 4, therefore, often concentrate on solving technical efficiency without worrying too much about asymmetric information.

The other categories certainly have their own superstars. It is not the intention of this paper to predict who will be the winner, nor is it even possible to do so. What we can say, in some limited and modest way, is that certain categories may lack a sustainable feature of the intrinsic transaction cost type that can be used as the driving edge in this competition for liquidity. Category 1 , for example, though very popular for people believing a notion of token economics in that if a

Table 1. A snapshot of crypto liquidity in 2017.

\begin{tabular}{ccc}
\hline \multirow{2}{*}{ Crypto } & \multicolumn{2}{c}{$2017 \mathrm{a}$} \\
\cline { 2 - 3 } & Liquidity (In Billions for Dec ) & Gain (\% over Dec previous year) \\
\hline RIPPLE & 60 & 36,018 \\
NEM & 2.7 & 29,842 \\
Ardor & 7.8 & 16,809 \\
Stellar & 5.8 & 14,441 \\
Dash & 7.8 & 9265 \\
Ethereum & 78 & 9162 \\
Golem & 3.5 & 8434 \\
Binance Coin & 1.6 & 8061 \\
Litecoin & 50 & 5046 \\
OmiseGo & 0.5 & 3315 \\
Bitcoin & N/A & 1318 \\
\hline
\end{tabular}

a. The figures fluctuate. According to CCN, total cryptocurrency market size in Oct 2018 is 210 billion. Source: https://www.slideshare.net/JonnyFry/an-analysis-of-icos-for-investors-and-public-listed-companies-by-jon ny-fry. 
platform can continuously reward digital wallets with increasing tokens, somehow people will like the tokens. This is building the technology mostly on featuring tokens having the third function of money, as a store of value. Our analysis in the previous section suggested that this strategy alone may not be sufficient to win, without showing their usefulness in other features of their currency, except by hoping that it will eventually become popular, and then people will use it to buy things.

There are certainly many companies in Category 2 that did well financially, if not in fiat money, but at least in terms of the cryptocurrency they mine. However, to have a miner that can build their expertise of mining all currencies seems unlikely. Without having expertise in mining all types, the success of the miner of a particular currency depends only on the success (liquidity and value of exchanging with fiat money) of the currency that is mined. This type of business is vulnerable to the liquidity risk of the currency.

Category 4, although goes beyond the mere motivation of store of value as in category 1 and 2, lacks the liquidity and volume category 3 can command. Thus, even though particular application in that category can address to the underlying needs of lowering of transaction costs of exchanges of real goods and services, e.g. Bitwage, ${ }^{6}$ its extensive adoption awaits the underlying global economics to develop in order to fully support it. Also, like any other platforms, it will be subject to competition.

The four categories of beachheads all use cryptocurrency as their units of account. Record keeping, however, goes beyond counting numbers, e.g. data collection, recording of news, medical records, land registration and real estate documents, criminal records, supply chain of products where the process and the origin of the products are important, e.g. IBM Trustchain tracking the origin of diamonds. ${ }^{7}$ In an op-ed piece by Henry Foley in Scientific American (April $11,2018)[15]$, the implications that wrong scientific research can have on the society in general is discussed. Blockchain technology can add accountability and visibility. For example, in clinical trials, this is exceedingly important because researchers/physicians have been reported to have produced fake consent forms. Consent forms are important in generating data for clinical trials, and they must seek prior acceptance of risks by the patients before the clinical trials are to be conducted.

The accounting/recording function of blockchain is now also extended to visuals, non-textual methods of expressions. For example, Sony recently filed for a patent to store users' digital data on the blockchain. Sony, once a pioneer in technology in sight and sound, created products such as Walkman and virtual reality headset PlayStation VR is now looking to manage and store users' digital entitlement data on the blockchain. There are now numerous possible implementations of DRM (Digital Rights Management) being explored by various companies [16]. 
All these are very important co-developing technologies, but they do not directly contribute to the liquidity for the cryptocurrency to promote it into a common medium of exchange, as they serve only niche markets. Yet, nobody would have guessed 30 years ago that music (serving only a niche market) could have driven us now into the lifestyle of using mobile phones to do everything. Our discussion of blockchain economics in previous section pointed us to look in the directions highlighted in this section nevertheless.

\section{Conclusions}

A cryptocurrency should not be founded based on pure greed or a get-rich-quick scheme. Bitcoin acquired its fame by its rapid (but volatile) increase in value. This may not be the best feature that will enable the sustainability of blockchain technology. High volatility can cripple investors' long run view of a particular cryptocurrency's usability as a store of value. What gave bitcoin that initial boost of attention as a medium of exchange is its built-in algorithm of a fixed supply-thus promising the luster of gold. Yet, striving to achieve interoperability across cryptocurrencies is likely to erode that emphasis. ${ }^{8}$ Pitching that as a scarce commodity like gold is unlikely to win. That, however, does not mean bitcoins will ever fall to a zero value, as many skeptics (including some economists cited earlier in this paper) have claimed. ${ }^{9}$

Our study shows that there are huge potentials to utilize blockchain technology. Still, it is one thing to say that a technology can do certain things that we are doing now; it's quite another to say that the technology can do it better. Competition between this new technology and the existing methods of doing things will continue for a long time. A couple of ATM bitcoin machines appearing sporadically retail certainly will not be sufficient for claiming success, the technology is still at a very early stage of adoption. We reasoned in this paper that the branding of a particular blockchain technology ultimately has to face the theoretical consideration not only for coding, but also simultaneously various considerations of cryptoeconomics. For the later, it includes both the macroeconomic problem of what is money, as well as the microeconomic problem of lowering transaction costs, particularly, adverse selection and moral hazards problems that can be managed by smart contracts. At a practical level, for any blockchain branding to be convincing/enticing beyond speculative motives, it is necessary to address to the fundamental economic values and problems that a particular technology is built to solve. Indeed, how the technology can do it better, rather than just rallying on a pitch line that "we can do it too".

Branding technologies, whether they are blockchains, or a substitute of blockchains, need to address its functionality (utility), on the type of industry

\footnotetext{
${ }^{8}$ Already, a so-called "layer two" is supposed to operate "on top" of existing blockchains, promising big strides in scalability, interoperability and functionality. https://www.coindesk.com/layer-2-blockchain-tech-even-bigger-deal-think/

${ }^{9}$ Ripple CEO, however, predicts that bitcoin price and XRP price will diverge.

https://www.ccn.com/ripple-ceo-garlinghouse-bitcoin-price-wont-correlate-with-xrp-price-forever/
} 
and people who will be using it, its usability in terms of UI/UX, and the type of extended support and verticals that the company will bring along. What started out as a technology that promised decentralization without middlemen has now also gradually evolved to become a tool for meeting compliance requirement. Dilemma of this sort needs to be resolved in order for a particular technology to be properly branded. In addition, other emerging technologies such as quantum computing, $5 \mathrm{G}$, etc. could trump the efficiency of whatever algorithm that a particular methodology is developing. Ultimately, it will be a race between technology and marketing. Without addressing to all these issues, a technology by itself cannot be branded to be disruptive, at least not yet.

\section{Conflicts of Interest}

The authors declare no conflicts of interest regarding the publication of this paper.

\section{References}

[1] Zhao, J.L., Fan, S. and Yan, J. (2016) Overview of Business Innovations and Research Opportunities in Blockchain and Introduction to the Special Issue. Financial Innovation, 2.

[2] Crosby, M.A, Pattanayak, P, Verma, S. and Kalyanaraman, V. (2016) Block Chain Technology: Beyond Bitcoin. Applied Innovation, No. 2, pp. 6-10.

[3] Underwood, S. (2016) Blockchain beyond Bitcoin. Communications of the ACM, 59, 15-17. https://doi.org/10.1145/2994581

[4] Mishkin, F.S. (2004) The Economics of Money and Financial Markets. 7th Edition, Pearson, Boston.

[5] Nofer, M., Gomber, P., Hinz, O. and Schiereck, D. (2017) Blockchain. Bus Inf Syst Eng, 59, 183. https://doi.org/10.1007/s12599-017-0467-3

[6] Jonker, N. (2018) What Drives Bitcoin Adoption by Retailers? Western Economic Association International Annual Conference, Vancouver, Canada, 26-30 June 2018. https://doi.org/10.2139/ssrn.3134404

[7] Allen, D. (1991) What Are Transaction Costs? Research in Law and Economics, 14, $1-18$.

[8] Akerlof, G.A. (1970) The Market for 'Lemons': Quality Uncertainty and the Market Mechanism. Quarterly Journal of Economics, 84, 488-500. https://doi.org/10.2307/1879431

[9] Mitnick, B. (2006) Origin of the Theory of Agency: An Account by One of the Theory $>$ Originators.

http://www.pitt.edu/ mitnick/agencytheory/agencytheoryoriginrev11806r.htm

[10] Coase, R. (1937) The Nature of the Firm. Economica, 4, 386-405. https://doi.org/10.1111/j.1468-0335.1937.tb00002.x

[11] Guarda, D. (2018) Blockchain + AI + Crypto Economics: Are We Creating a Code Tsunami? The Future of Blockchain, Vancouver, 8 May 2018.

[12] Raymond, E.S. (2009) The Cathedral and the Bazaar. Internet Version, O'Reilly Media.

[13] Moore, G. (1991) Crossing the Chasm. Harper Business Essentials. 
[14] Lorne, F., et al. (2018) Thinking Framework for Blockchain Branding. NYIT Working Paper.

[15] Foley, C.H. (2018) Many Scientific Studies Are Bogus, but Blockchain Can Help. https://blogs.scientificamerican.com/observations/many-scientific-studies-are-bogu s-but-blockchain-can-help/

[16] Terzo, G. (2018) Sony Files Patent for Digital Rights Storage on a Blockchain. https://www.ccn.com/sony-files-for-blockchain-fueled-drm-patent/ 